\title{
A new approach to occupational reintegration using the hybrid assistive limb voluntary driven exoskeleton HAL
}

\author{
Hans-Jörg Piasecki \\ German Social Accident Insurance, Germany. hans-joerg.piasecki@bgrci.de \\ Oliver Cruciger \\ BG University Hospital Bergmannsheil Bochum, Germany. oliver.cruciger@bergmannsheil.de
}

The socio-professional reintegration of spinal cord injured (SCI) patients, remains challenging due to aggravating circumstances. In spite of conventional treatment the patients' limitations regarding mobility, chronic pain syndrome, incontinence and prolonged inability to work due to decubitus ulcer and contractures often add up to an insurmountable disadvantage in the occupational rehabilitation. An international cooperation between Cyberdyne Inc. (Japan) and the professional association of raw materials and chemical industry (Germany), focusing on a new therapeutic approach reveals promising outcomes to improve the situation of these spinal cord injured patients. During the treatment the patients perform a locomotion training using the hybrid assistive limb (HAL $\left.{ }^{\circledR}\right)$ exoskeleton which offers the unique possibility to record and amplify minimal bioelectrical signals by emg-electrodes on the skin of the lower extremities resulting in machine supported gait pattern synchronously to the patient's voluntary drive. After 12 weeks of training significant improvements, regarding functional mobility has been achieved leading overall to a more autonomous and motivated patient. The presentation of the results and potential impact on the occupational rehabilitation will be based on video supported case reports. 